\section{Application of teledentistry in oral medicine in a Community Dental Service, N. Ireland}

\author{
M. Bradley, ${ }_{1}^{1}$ P. Black, ${ }_{1}^{2}$ S. Noble, ${ }^{3}$ R. Thompson ${ }^{4}$ and P. J. Lamey ${ }^{5}$ \\ VERIFIABLE CPD PAPER
}

IN BRIEF

- Presents a pilot series of patients with oral mucosal problems who had high quality clinical photography of their lesions taken in primary care and then viewed online by an oral medicine specialist.

- Distance diagnosis of disease has the potential to significantly alter patient referrals.

- Most participants could be managed in primary care without attending a hospital specialist.

Currently, patients with oral medicine conditions from all areas of Northern Ireland are referred by dentists and doctors to a small number of specialist services: predominantly, the Regional Oral Medicine Consultant at the School of Dentistry, Belfast. On receipt of the referral the consultant makes an assessment of the urgency of the case and the patient is placed on a waiting list. Until the recent implementation of waiting list initiatives (Elective Access Protocol, Department of Health, N. Ireland, 2006), patients remained on the waiting list for long periods of time. Analysis of these patient profiles highlights that many need both multiple treatment and review appointments of their chronic conditions, and consequently remain in the hospital system for significant periods of time. This increases the waiting time for these services. The idea of using teledentistry to triage referrals, and its potential as a tool to support locally based treatment, poses an alternative approach to the management of oral medicine referrals. It may be of particular interest to practitioners in rural locations where distance from the regional centre is significant. In 2005, to test this theory, a prototype teledentistry system was set up as part of a service improvement scheme by the Community Dental Service of the Homefirst Legacy Trust (now Northern Trust) in partnership with the Oral Medicine Department at the School of Dentistry, Royal Group of Hospitals Legacy Trust (now Belfast Trust). This paper describes the feasibility study.

\section{INTRODUCTION AND BACKGROUND}

Teledentistry is defined as "the provision of real time and offline dental care such as diagnosis, treatment planning, consulting and follow up via electronic transmission from different sites.' ${ }^{1}$ This technique is similar to telemedicine, which was first used in the 1970s by NASA ${ }^{2,3}$ (National Aeronautical and Space Administration), and more recently by the US Military. ${ }^{4,5}$ Teleradiology and teledermatology are still among the most common applications. ${ }^{6}$ Two main types of technology are used: (i) live consultations, or videoconferencing,

\footnotetext{
${ }^{1 *}$ Senior Community Dentist, Gerodontology, ${ }^{3}$ Clinical Director, Community Dental Service, Northern Trust, Community Dental Dept, Spruce Hse, Braid Valley Site, Cushendall Rd, Ballymena, Co Antrim; ${ }^{2}$ Network and Security Manager, ICT Dept, Northern Trust Headquarters, The Cottage, Greenmount Ave, Ballymena, Co Antrim; ${ }^{4}$ Chief Dental Technical Officer, Dept of Prosthetic Dentistry, ${ }^{5}$ Professor of Oral Medicine, School of Dentistry, Belfast Trust, Royal Victoria Hospital, Grosvenor Rd, Belfast;

*Correspondence to: Ms Maeve Bradley

Email:maeve.bradley@northerntrust.hscni.net
}

\section{Refereed Paper}

Accepted 14 June 2010

DOI: $10.1038 /$ sj.bdj.2010.928

${ }^{\circledR}$ British Dental Journal 2009; 209: 399-404 which are described as resource and cost intensive; and (ii) asynchronous, or store and forward consultations, which are considered to have the potential to be cost effective. Although use of teledentistry is uncommon in dentistry in UK, increasingly dental practitioners and hospital specialists are seeking opinions on digital images of oral lesions. ${ }^{7}$ This is the aspect with which this service improvement was concerned. In California, USA, Youngai and Messadi ${ }^{8}$ described a pilot study examining the reliability and accuracy of diagnosis of oral mucosal disease based on written information, without any visual images of the lesion. The diagnostic accuracy was found to be moderate. In the absence of reported teledentistry studies, 'teledermatology' was proposed as a reasonable comparator. Gilmour et al. ${ }^{9}$ report the diagnostic accuracy of teledermatalogy, using a captured image rather than a face to face examination, as between 57\% and 83\%. Furthermore they estimated the cost of a teledermatology consultation and follow up appointment with a primary care practitioner as $37 \%$ lower than a face to face consultation with the hospital specialist. Therefore it was suggested that teledentistry might yield similar cost benefits. Despite this frequently quoted potential benefit, and its ability to provide more equitable access to care, this technology has not been adopted into dental practice. Scully et $a .^{7}$ suggest that this reluctance may be related to possible medico-legal complications. Experience drawn from countries like the USA describe the difficulties associated with practitioner licensing regulations which limits practice to within one state. ${ }^{10,11}$ Where the same practitioner gives advice about patients in another state this is deemed illegal. In response, by 2002, some changes in US legislation had occurred. Medicare, one of the leading health insurance companies in the USA, now covers use of telemedicine in restricted treatment areas, thus acknowledging their acceptance of telemedicine practice as a legitimate way of providing health care services. ${ }^{6,12}$

In November 2008 the UK society Dental Protection published a position paper on teledentistry warning of some of the 
potential legal pitfalls associated with its use especially when applications crossed jurisdictional or national boundaries. ${ }^{13}$ That position paper highlights the more stringent requirements of the consent process, management of patient information, and potential for malpractice recommending use of a written protocol.

Nevertheless, there is still a drive within the NHS to use technology creatively in the provision of health care, and indeed in 2008 the European Centre for Connected Health was established in Northern Ireland which aims to investigate innovative approaches to management of health care involving technology, like teledentistry. ${ }^{14}$ In addition there is a persistent nationwide call for the introduction of electronic patient health records as utilised in this teledentistry prototype. ${ }^{15}$

The most frequently cited factor in support of the adoption of 'teledentistry' is its potential as a more cost efficient way of providing services. As yet there is little evidence to support this. More surprisingly the authors of a recent 2002 systematic review investigating the cost effectiveness of telemedicine interventions concluded that again there was a lack of good quality evidence to support this concept.,16 of an initial 612 telemedicine studies identified only 55 articles met the agreed parameters; most were from the USA (60\%), others came from Norway, Australia, Canada, Europe, and Japan. Obvious by its omission from this list is the UK: well designed research into the cost effectiveness of telemedicine and teledentistry is still required in the UK.

\section{UK teledentistry research}

Distance from referral site has been previously highlighted as an important restrictive factor influencing the likelihood of referral. ${ }^{17,18}$

Nuttall et al. ${ }^{19}$ examined the feasibility of using teledentistry in provision of specialist restorative care in the Highlands and Islands of Scotland (HIT). They established that access to, and equity of access, to consultant led restorative services in rural Scotland was poor. This negatively influenced the numbers of patients referred by dentists to secondary care. The conclusion of an economic analysis of this HIT teledentistry project was that the greatest benefits, and biggest cost savings, were recouped where patients had to travel long distances to visit the hospital consultant. ${ }^{20}$ It was interesting to note that these teleconsultations which utilised videoconferencing were found to be more expensive than outreach visits by the consultant. It should be remembered that videoconferencing is the more costly means of providing teledentistry services, and the associated costs may have decreased in the nine years since that study was conducted. That research also demonstrated that using teledentistry can reduce health care inequalities, giving people better and fairer access to specialist dental services.

In the speciality of orthodontics there have been a number of reports in the literature examining the use of teledentistry. In 2002 Stephens et al. ${ }^{21}$ describe the 'Teledent Southwest' study which investigated if teledentistry could be used as the method of providing consultant orthodontic advice to dentists for a group of 163 patients. As a preliminary to their study, the consultant orthodontists tested their reliability/diagnostic accuracy both face to face and using teledentistry and concluded that it was satisfactory. Although dentists' feedback on this application was good, this was not reflected in increased usage of the teledentistry system during and after the study. This was attributed to the lengthy patient "work up" required for what was regarded as a non-urgent referral, and for which there was no remuneration for the dentist. The greatest benefit was found to be a reduction in the number of 'inappropriate referrals', largely referring to the timing of the referral. This could have a dramatic effect on consultant orthodontic waiting lists.

Similar results were found by Mandall et $a l .{ }^{22}$ in 2005 when investigating the use of teledentistry in screening 327 new patient orthodontic referrals using store and forward technology.

In his follow up survey of over 200 dentists, most were found to be supportive of using a store and forward teledentistry system for new patient orthodontic referrals. ${ }^{23}$ However, the same financial concerns were raised by dentists as had been voiced in the Teledentistry South West study. More recently, in 2007, Bradley et al. ${ }^{24}$ found that just under half (46\%) of 91 dental practices in West Yorkshire were supportive of the use of online (teledentistry) orthodontic services. This response might reflect the fact that only 39\% had access to the Internet in their practices, a prerequisite for using teledentistry. Even so this figure of 39\% represents a significant improvement from the picture in 2000, when in a small study of oral medicine referrals to Birmingham Dental School it was found that the majority of dentists had neither Internet access, nor fax machines. ${ }^{25}$ This limited transmission of urgent referrals to specialist services by telephone or post. In that study, one third of the group of dentists felt that a description of the patient's oral pathology was not required in the referral letter, and although use of clinical photography was cited as a solution, it was dismissed as being too time consuming. A digital solution was not suggested. Another aspect of that study related to an assessment of the quality of referral information received by the Hospital Oral Medicine Service. The authors concluded that the dentists in the study gave insufficient information to allow effective prioritisation of patients with oral cancer. This is not unusual as McLeod et al. ${ }^{26}$ reported similar findings when examining the cause of delays in oral cancer diagnosis. In 2000 in an effort to improve this situation the Department of Health set out a two week waiting time target for a diagnosis of cancer where it has been suspected by a primary care professional. ${ }^{27}$

\section{METHODOLOGY: AIMS AND OBJECTIVES}

The aims of this service improvement were:

- To install a prototype teledentistry system in Antrim Community Dental Clinic (thereafter 'teledentistry site') linked to the School of Dentistry Belfast. This necessitated sourcing all software and hardware components required, as there was no comparable dental system established in the UK or Republic of Ireland

- To undertake a six month study (January-June 2006) to assess the feasibility of using teledentistry to process oral medicine referrals to a Belfast hospital consultant. This involved patient assessment by the lead community clinician, remote from the consultant. Clinical photography was used to supplement a patient 


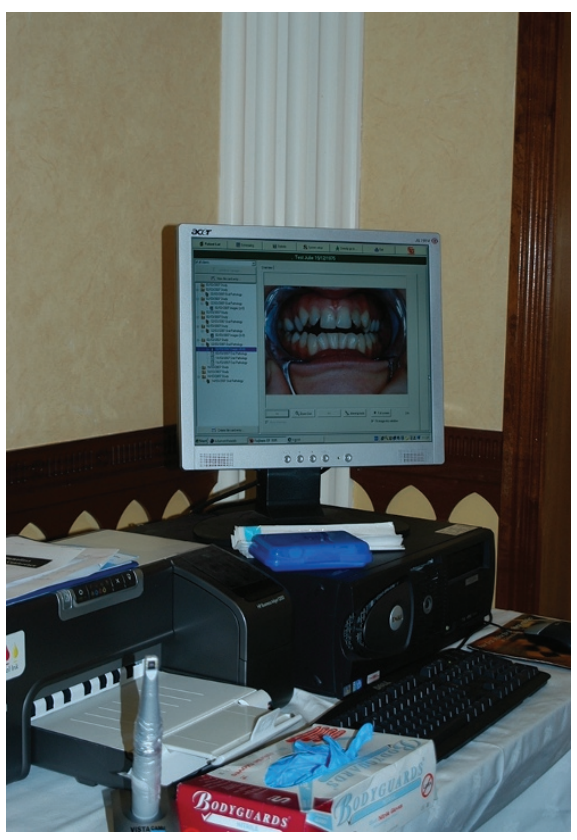

Fig. 1 Teledentistry set up showing an intra oral camera in the foreground and including a clinical image

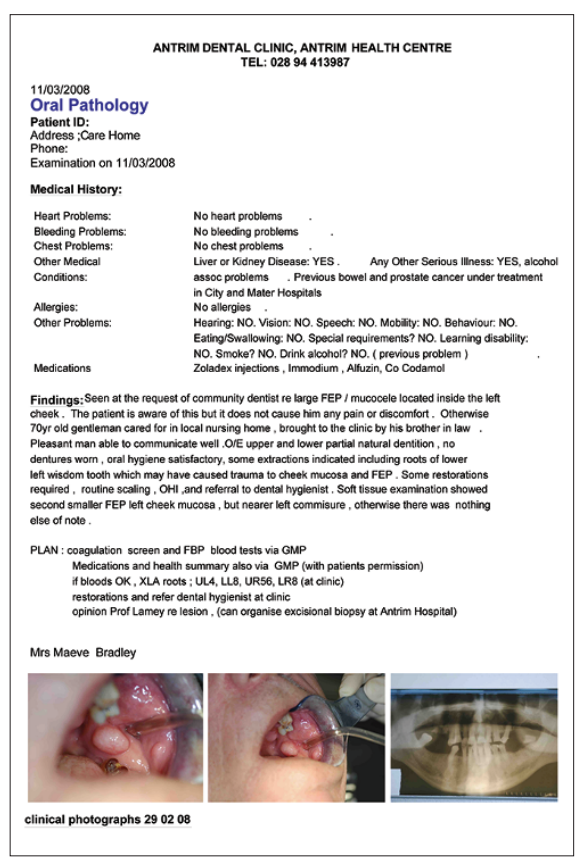

Fig. 2 Standard information collected for each patient including medical and dental history, clinical photography and radiograph

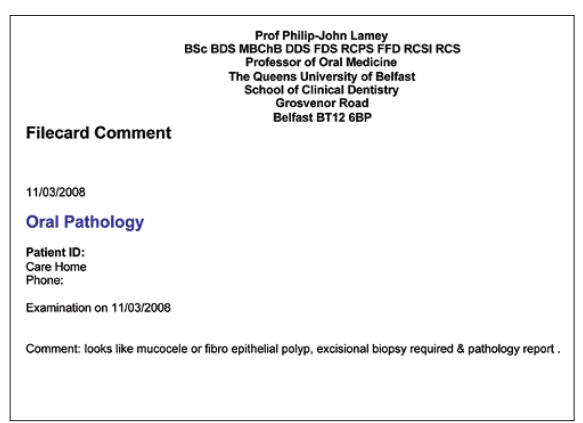

Fig. 3 Consultant opinion in relation to oral mucosal lesion in Figure 2 electronic referral viewed by the Belfast Consultant using the HPSS net. This replaced the traditional referral pathway whereby patients travelled to see the hospital consultant.

Technical parameters including use of an electronic record and suitability of an associated data communication system were specific areas of interest. In addition a preliminary assessment of patient and clinician acceptability of the teledentistry experience was identified as another facet for exploration.

\section{Setting}

The Northern Health and Social Care Trust is the second largest trust in N. Ireland with a population of approximately 450,000 . It occupies predominantly the north of the province and comprises both urban and rural communities. The prototype teledentistry system was installed in the Community Dental Clinic, in Antrim, a small town 17 miles from Belfast .

\section{Study design}

A sample of 41 patients were recruited to the feasibility study. Most were assessed at the agreed teledentistry site but in cases where patients were unable to travel, a domiciliary assessment was arranged. Twenty patients requiring an oral medicine assessment were under the care of community dentists in the Homefirst Legacy Trust. The other 21 were patients on the Oral Medicine Consultant's Hospital waiting list in the School of Dentistry who lived within the Northern Trust Area. The latter group were recruited to the study by letter and were invited to attend the teledentistry site where the CDS lead clinician undertook a one visit 'teledentistry assessment' (Fig. 1). Standardised information was agreed for this purpose which included medical information, pathology parameters, supplemented by digital photographs, video footage and X-rays.

Following the patient assessment computerised/electronic patient reports were compiled by the lead CDS clinician at the teledentistry site (Fig. 2). Using the teledentistry system these were communicated to the consultant at Belfast for his opinion using the HPSSnet, a secure version of the Internet. In response the consultant used the teledentistry system to relay his decisions about the patient's diagnosis to the teledentistry assessment centre (Fig. 3). Hard copies of the initial electronic patient report and consultant response were printed and filed in the patient notes at the assessment clinic in case of system error. These were also forwarded to other clinicians involved in the patients' care, eg the doctor, where appropriate.

On completion of all patient assessments the CDS clinician met with the consultant in Belfast to check the integrity of the electronic patient records transmitted over the course of the study.

\section{Ethical considerations}

A protocol for the conduct of the feasibility study was agreed. A written consent form with explanatory information was designed for participating patients. Access to the teledentistry system was restricted to two users: the consultant at Belfast and the CDS lead clinician. To ensure patient confidentiality, patients were allocated unique identifier numbers, patient data was encrypted and clinicians were allocated system passwords. A back up system for the patient data was also installed at the assessment clinic.

Patients who did not wish to participate in the teledentistry feasibility study were not disadvantaged and their referrals were processed in the normal way.

\section{Training}

Before the feasibility study competency based oral medicine update training was provided by the Consultant in Oral Medicine at the School of Dentistry Belfast for the CDS lead clinician. The training period involved weekly sessions over the course of a year. In addition specialist support in use of digital photography was provided by the School of Dentistry. The suppliers of the software program provided training and technical support for both clinicians for the duration of the feasibility study.

\section{Participants}

Of the 21 hospital waiting list 17 patients participated in the study. In this group the average patient age was 55.5 years and the majority were able to express their symptoms. In 26\% of cases the original source of referral was from the family doctor, $69 \%$ from the family dentist, and in $4.3 \%$ of cases from a consultant oral surgeon. 
Most (79\%) of the 20 community dental service patients were resident in nursing homes; 75\% required a domiciliary assessment. The average age of this group of participants was 79.6 years, and just under half were over 81 years old. In a significant proportion of these cases the oral pathology was identified by one of the community dentists while undertaking a dental screening. Approximately one third of this group were not capable of consenting for their dental treatment (Table 1 )

\section{OUTCOME MEASURES}

1. The main outcome measure was

to develop and set up a prototype teledentistry system to support triage and management of oral medicine referrals which demonstrated robust technical performance

2. A secondary outcome related to an analysis of the characteristics of the 37 participants of the feasibility study; specifically the provisional diagnosis and treatment priority allocated as a result of the teledentistry assessment.

\section{RESULTS}

\section{Electronic referral system}

All of the electronic referrals were retrieved by the Belfast Consultant without error. The CDS clinician quality assured all referrals by visiting the Belfast site.

\section{Data communications}

The teledentistry system operates on the Trust's network with a broadband connection to the School of Dentistry Belfast. It was decided to use a dedicated link to the School of Dentistry to avoid any potential problems during the pilot phase.

While all of the electronic referrals and high resolution clinical photographs were viewed satisfactorily by the Belfast Consultant; a small percentage of the patient's video clips were problematic.

\section{System integrity}

The system has been operational since January 2006; to date all patient data have remained confidential. A back up system was installed at the assessment centre in case of system error. Hard copies of each patient's report were made for the Belfast Consultant, and for the assessment centre file. Where appropriate and with the

\begin{tabular}{|c|c|c|c|}
\hline Age group & Hospital group & CDS group & Total \\
\hline Under 60 years & 7 & 2 & 9 \\
\hline $61-80$ years & 9 & 9 & 18 \\
\hline $81+$ years & 1 & 9 & 10 \\
\hline Total & 17 & 20 & 37 \\
\hline
\end{tabular}

\begin{tabular}{|c|c|c|c|c|}
\hline & $\begin{array}{l}\text { Hospital waiting } \\
\text { list participants }\end{array}$ & CDS participants & $\begin{array}{l}\text { Total } \\
\text { number }\end{array}$ & $\begin{array}{l}\text { Number } \\
(\%)\end{array}$ \\
\hline DNAd appt & 4 & - & 4 & $4 / 41(10 \%)$ \\
\hline Referred to wrong speciality & 1 & & 1 & $1 / 41(2.5 \%)$ \\
\hline Harmless lesion - discharged & 1 & - & 1 & $1 / 41(2.5 \%)$ \\
\hline $\begin{array}{l}\text { Could be managed in CDS } \\
\text { with supervision }\end{array}$ & 11 & 16 & 27 & $27 / 41(65 \%)$ \\
\hline Require urgent hosp treatment & 4 & 4 & 8 & $8 / 41(19 \%)$ \\
\hline Total & 21 & 20 & 41 & $100 \%$ \\
\hline
\end{tabular}

patient's permission other copies have been forwarded to clinical nurse managers at nursing homes, and family doctors for their information.

\section{Patient and clinician acceptability}

In total 90\% (37/41) of those invited participated in the study. None withheld their consent for either digital photography, or video footage of their oral mucosal pathology. All participants used the opportunity to view their personal video, at the end of the assessment appointment. All patient electronic records were retrieved by the consultant in Belfast without error. He judged the quality of the digital images transmitted as satisfactory and in the majority of cases, when supported by the agreed medical and dental information, of sufficient quality to facilitate his provisional diagnosis of the patient's oral mucosal disease. In summary the technical aspects of the teledentistry system were satisfactory.

\section{Procedural experience}

A software program was identified in September 2005 by the Homefirst legacy Trust ICT manager. The software solution called ADAM (Advanced Digitalisation and More) is manufactured by Fujinon, Germany for use in medical endoscopy. It was installed with associated hardware in the teledentistry site.

\section{Oral mucosal presentations}

Overall 65\% (27/41) of patients had common oral mucosal diseases including: candida, ulceration, tongue lesions, fibro epithelial polyps, mucoceles, amalgam tattoos, denture granulomas and keratosis. The consultant concluded that preliminary treatment of this group of patients could be provided in the community dental service with his distant supervision using agreed hospital protocols via the teledentistry system. He evaluated that eight (20\%) patients required urgent hospital treatment: six for biopsy of what looked like sinister lesions or potential carcinomas and two for other uncommon conditions that required his expertise, including one case of orofacial granulomatosis (OFG) and one of sialosis. In addition one patient had been referred to the wrong dental speciality, and another had been referred with a harmless lesion that did not require assessment or treatment (Table 2).

\section{DISCUSSION}

\section{Referral information}

In this study, the quality and quantity of patient information provided was variable between doctors and dentists. Both professionals included different types of information: doctors provided better medical information about the patient being referred, whereas dentists largely did not. 
A small number of patients were referred to the consultant without their knowledge (or consent), and in a few cases the urgency of the referral was not indicated. The literature supports the notion that poor quality of referral letters is associated with delayed 'first outpatient assessments. ${ }^{25,26}$ This could result in delayed oral cancer diagnosis, associated treatment and less favourable outcomes for the patient. Use of a standardised/agreed referral format is therefore recommended and should be facilitated and encouraged within primary care. Furthermore this study has shown that incorporating high resolution digital images along with agreed medical and dental information facilitates prioritisation of those requiring urgent assessment. Those who do not need a consultant referral or who have been inappropriately referred can also be easily identified. This process described as 'waiting list triage' appears to be a strong feature of the teledentistry system. By using teledentistry in this way, validation of and reduction in waiting lists could be achieved and assurance that patients with potentially sinister lesions are more likely to be prioritised for consultant assessment early. In this way UK cancer targets for first outpatient assessment could be achieved.

\section{Benefits to elderly}

An unexpected benefit of this service development was successful community based patient management of a number of elderly patients. This was only possible with the distant supervision of the consultant who assured consistency of approach, assessed urgency/priority, assisted with diagnosis, and agreed appropriate treatment. He was available for further advice when required and where there were concerns the patient could be re-referred. As a result the majority (65\%) of the community group of patients avoided hospital based treatment entirely. By providing locally based assessment the need for expensive transport was avoided, accompanying staff time was significantly reduced, and patient anxiety was alleviated. Indeed when the patient was particularly frail, confused or sick s/he was best served when the preliminary diagnosis, and information gathering was undertaken on a domiciliary basis (75\%). Increasingly more adults are living longer and to older ages. Currently there are 104 residential and nursing homes in the NHSCT. ${ }^{28}$ A predicted increase of $25 \%$ in the number of elderly adults residing in NHSCT Trust Area is expected by 2015 , from the 2005 figure of 61,690 to $77,275 .{ }^{29}$ This is largely in keeping with the $20 \%$ expected increase in the older population in the province of $\mathrm{N}$. Ireland by $2015^{30}$ and with the UK predicted increase of 19\% for this subgroup of the population by $2020 .^{31}$ In the future, the implications for service needs for this cohort of the population are likely to be significant, and the potential of teledentistry as an alternative means of delivering oral medicine services should be considered in future service planning.

\section{Cost effectiveness}

The capital costs associated with setting up the system were approx $£ 25,000$ (2006/07). A comparison of the cost of providing a teledentistry assessment within the community dental service undertaken by a trained CDS clinician, as an alternative to an assessment provided by the consultant in hospital, is not known. This complex analysis, which could provide the basis for future research, must include measurement of quantitative components such as skill mix, transport, distance, and clinician's time. Equally other less easily measured qualitative aspects which relate to the patient's experience of the care provided must also be included ie associated reduced patient anxiety, potential to give patients a provisional diagnosis quickly, the opportunity to be in a familiar environment either at a local clinic or in their place of residence.

\section{Limitations}

As this was a small study $(n=41)$ the results should be interpreted with caution. In this study the participants $(n=41)$ had one assessment/diagnostic appointment with the lead CDS clinician at the community clinic to construct an electronic patient record, which included clinical photography of the lesion. As this assessment was not repeated as a 'face to face' diagnostic appointment with the consultant, his diagnosis was based on the clinical photography provided by the CDS clinician in the electronic record. There was no opportunity to compare this provisional diagnosis with the provisional diagnosis he would assign to the same patient at a face to face appointment. Thus, a more rigorous study designed to examine the validity of using teledentistry to diagnose oral mucosal disease is required before firm conclusions can be made, or this method adopted more fully. Diagnosing by 'visual examination only' (either face to face, or from digital photography) has limitations even when supported by appropriate medical and dental information. Before a definitive diagnosis can be made an appropriate diagnostic test (often biopsy) may be required. As some oral mucosal lesions have the potential to become malignant over the course of time while biopsy may not be initially indicated, it may become so at a later presentation. Clinical experience is key to making decisions about which lesion, and at what time, a biopsy (or other diagnostic test) is indicated. In this study a 'successful' teledentistry diagnosis was 'agreed" when the patient's lesion responded favourably to the treatment provided, ie significantly improved, or resolved entirely. This approach is consistent with the hospital consultant's current practice.

\section{CONCLUSIONS AND RECOMMENDATIONS}

Setting aside the governance issues associated with using a teledentistry system and despite the limitations described, this study demonstrates that using teledentistry in the management of patients with oral mucosal disease can work successfully. It is especially suitable for management of referrals of older dependant adults who have oral mucosal disease

\section{Recommendations}

1. A standardised referral form should be used for oral medicine referrals by all primary health care professionals, including doctors

2. Dental professionals should be encouraged to take clinical photographs of oral pathology routinely (with the patient's consent) as this enhances both the quality of referrals and patient clinical records alike

3. Research aimed at examining the potential of using clinical photography communicated by a teledentistry system as a diagnostic tool for patients with oral mucosal disease as an alternative to the face to 
face consultant consultation should be undertaken

4. Research aimed at evaluating the cost effectiveness of using a community based teledentistry service for the management of oral mucosal disease, in comparison to the traditional consultant led hospital based approach, should be undertaken.

The authors would like to acknowledge the sup port of Hospital Services Ltd, Belfast, who for the duration of the feasibility study, provided both the 'ADAM' software program, and training in its use. Thanks must also go to the members of the original inter-trust teledentistry steering group from both legacy trusts specifically: Mrs Hazel Baird, Director of Nursing Dental and Governance Directorate, Homefirst Legacy Trust and Dr Mike Mannion Medical Director, Homefirst Legacy Trust. Latterly to Mrs Margaret Gordon, Assistant Director, Women and Children's Directorate, NHSCT. Final acknowledgements to Dr Ciaran Shannon, Consultant Clinical Psychologist, Queen's University, Belfast, and Professor Gerry Linden, School of Dentistry Belfast for assistance in writing this paper.

1. Chen J, Hobdell M, Dunn K, Johnston K, Zhang J. Teledentistry and its use in dental education. J Am Dent Assoc 2003; 134: 342-346.

2. Brown N. A brief history of telemedicine. 30 May 1995 (Available online: http://tie.telemed.org/ articles/article.asp?path=articlesftarticle=tmhisto ry_nb_tie95.xml).

3. Currell R, Urquhart C, Wainwright P, Lewis R. Telemedicine versus face to face patient care: effects on professional practice and health care outcomes. Cochrane Database Syst Rev 2000; CD002098.

4. Clark G. Teledentistry: what it is now, and what it will be tomorrow? J Calif Dent Assoc 2000: 28: 121-127.

5. Folke L. Teledentistry. An overview. Tex Dent J 2001;
118: $10-18$

6. Hartvigsen G, Johansen M A, Hasvold P, Bellika J G et al. Challenges in telemedicine and eHealth: lessons learned from 20 years with telemedicine in Troms $\varnothing$. Stud Health Technol Inform 2007; 129: 82-86.

7. Scully C, Porter S. Referrals in oral medicine. Dent Update 2007; 34: 340-350

8. Youngai F, Messedi D. E-mail-based oral medicine consultation. J Calif Dental Assoc 2000; 28: 144-151.

9. Gilmour E, Campbell S M, Loane M A, Esmail A et al. Comparison of teleconsultations and face-to-face consultations: preliminary results of a UK multicentre teledermatology study. Br J Dermatol 1998; 139: $81-87$

10. Sfikas P. Teledentistry: legal and regulatory issues explored. J Am Dent Assoc 1997; 128: 1716-1718.

11. Golder D T, Brennan K A. Practicing dentistry in the age of telemedicine. JAm Dent Assoc 2000; 131: 734-744.

12. Whitten PS, Richardson J D. A scientific approach to the assessment of telemedicine acceptance. J Telemed Telecare 2002; 8: 246-248.

13. Teledentistry, a position paper. Dental Protection Limited, November 2008. (Available online http://www.dentalprotection.org/uk/news/ positionstatements/teledentistry.).

14. European Connected Health Expo Day, May 2008 (Letter.).

15. Reynolds P A, Harper J, Dunne S. Better informed in clinical practice - a brief overview of denta informatics. Br Dent J 2008; 204: 313-317.

16. Whitten PS, Mair FS, Haycox A, May C R et al. Systematic review of cost effectiveness studies of telemedicine interventions. BMJ 2002; 324: 1434-1437.

17. Linden G. Variation in periodontal referral by general dental practitioners. J Clin Periodontol 1998; 25: 655-661.

18. Coulthard P, Kazakoul, Koran R, Worthington $\mathrm{H}$. Referral patterns and the referral system for oral surgery care. Part 2: The referral system and telemedicine. Br Dent J 2000; 188: 388-391.

19. Nuttall N M, Steed M S, Donachie M A. Referral for secondary restorative dental care in rural and urban areas of Scotland; findings from the Highlands and Islands teledentistry project. Br Dent J 2002; 192: 224-228.

20. Scuffhan P A, Steed M. An economic evaluation of the Highlands and Islands teledentistry project. J Telemed Telecare 2002; 8: 165-167.

21. Stephens $C$, Cook J, Mullings C. Orthodontic referrals via Teledent Southwest. Dent Clin North Am 2002; 46: 507-520.

22. Mandall N A, O'Brien K D, Brady J, Worthington $H V_{\text {, }}$ Harvey L. Teledentistry for screening new patient referrals. Part 1: A randomised controlled trial. $\mathrm{Br}$ Dent J 2005 ; 199: 659-662.

23. Mandall N, Quereshi U, Harvey L. Teledentistry for screening new patient referrals. Part 2: GDP perception of the referral system. Br Dent J 2005; 199: 727-729

24. Bradley S, Williams S, D'Cruz J, Vania A. Profiling the interest of GDPs in West Yorkshire in using teledentistry to obtain advice from orthodontic consultants. Prim Dent Care 2007; 14: 117-122.

25. White D A, Morris A J, Burgess L, Hamburger J Hamburger R. Facilitators and barriers to improving the quality of referrals for potential oral cancer. Br Dent J 2004; 197: 537-540.

26. McLeod N M, Saeed N R, Ali E A. Oral cancer: delays in referral and diagnosis persist. Br Dent J 2005; 198: 681-684.

27. Department of Health. Referral guidelines for suspected cancer. NHS Executive. 13 April 2000. (Available online: www.dh.gov.uk).

28. Source: The Regulation and Quality Improvement Authority, Northern Ireland (ROIA). (Available online www.rqia.org.uk).

29. Living Well Ageing Better. A Strategy for Services for Older People 2006-2011. Northern Health and Social Care Trust (report). (Available online http:// www.northerntrust.hscni.net/pdf/Living_well_ageing_better.pdf).

30. Northern Ireland Statistics and Research Agenc, Mid-year Population Estimates. (Available online http://www.nisra.gov.uk/archive/ demography/population/mid-year/NI_Home_ Pop_5yrolds(1961-2008).xIs).

31. Oral healthcare for older people, 2020 vision. A BDA Key Policy Paper, May 2003. 